Supporting Information

\title{
Facile Preparation of Well-Defined Uniform Hydrophilic Hairy Hollow Functional Polymer Micro- and Nanoparticles
}

Congguang Zheng†, Yan Zhou†, Huiqi Zhang*

State Key Laboratory of Medicinal Chemical Biology, Key Laboratory of Functional Polymer Materials (Ministry of Education), Collaborative Innovation Center of Chemical Science and Engineering (Tianjin), and College of Chemistry, Nankai University, Tianjin 300071, P. R. China. E-mail: zhanghuiqi@nankai.edu.cn.

\section{Preparation of "Living" PMAA Microspheres with Surface-Bound Dithioester Groups}

(Entry 1 in Table 1). "Living" PMAA microspheres with surface-bound dithioester groups were prepared via RAFTPP following our previous method (Macromolecules 2019, 52, 143156). MAA(3.82 mL, $44.82 \mathrm{mmol}), \mathrm{CDB}(59.89 \mathrm{mg}, 219.84 \mu \mathrm{mol})$, dried acetonitrile (120 mL), and AIBN $(5.22 \mathrm{mg}, 31.79 \mu \mathrm{mol})$ were added into a one-neck round-bottom flask $(250 \mathrm{~mL})$ with an oval-shaped magnetic stirring bar (with a length of $3.0 \mathrm{~cm}$ ) inside successively. The resulting solution was then purged with argon for $30 \mathrm{~min}$ in an ice-water bath, sealed, immersed into a thermostated oil bath at $60{ }^{\circ} \mathrm{C}$, and stirred for $24 \mathrm{~h}(200 \mathrm{rpm})$. The polymer product was collected by centrifugation and subsequently washed with acetonitrile thrice. After being dried at $40{ }^{\circ} \mathrm{C}$ under vacuum to the constant weight, a light pink powder was obtained (yield: $25 \%$ ).

To obtain enough "living" PMAA microspheres for this study, the above polymerization was repeated several times and the obtained products were mixed together for the following use.

In this context, it is worth noting that the yields of "living" PMAA microspheres increased with increasing the polymerization time and they could range from $3 \%$ to $55 \%$ by changing the polymerization time from 6 to $48 \mathrm{~h}$. We chose a polymerization system with a PMAA particle yield of $25 \%$ here just to demonstrate the proof-of-principle. In addition, the excellent repetition of the RAFTPP of MAA and its upscaling possibility could also lead to enough "living" PMAA particles easily either by combining the products of several batches or upscaling the polymerization system. Furthermore, about $1 \mathrm{~g}$ of "living" PMAA particles could be produced in each batch (as described in this work), which is already quite efficient for many applications. 


\section{Preparation of "Living" Core-Shell-Structured Polymer Microspheres Including PMAA@P(MAA-co-AnHEMA-co-EGDMA) (Entry 6 in Table 1), PMAA@P(MAA-co- BAC) (Entry 7 in Table 1), and PMAA@P(NIPAAm-co-MBA) (Entry 8 in Table 1).}

PMAA@P(MAA-co-AnHEMA-co-EGDMA) microspheres (entry 6 in Table 1) were prepared as follows: To a one-neck round-bottom flask $(100 \mathrm{~mL})$ were added "living" PMAA microspheres $(60.50 \mathrm{mg})$ and dried acetonitrile $(60 \mathrm{~mL})$. After the above mixture was ultrasonically dispersed at room temperature for $2 \mathrm{~min}$, AnHEMA (10.82 mg, $32.36 \mu \mathrm{mol})$, MAA $(27 \mu \mathrm{L}, 0.32 \mathrm{mmol})$, EGDMA $(120 \mu \mathrm{L}, 0.64 \mathrm{mmol})$, and AIBN (0.42 mg, $2.56 \mu \mathrm{mol})$ were added successively. The reaction mixture was then degassed by bubbling argon for $30 \mathrm{~min}$ in an ice-water bath, sealed, immersed into a thermostated oil bath at $60{ }^{\circ} \mathrm{C}$, and magnetically stirred for $24 \mathrm{~h}$. After centrifugation, the resulting solid product was thoroughly washed with acetonitrile and dried at $40{ }^{\circ} \mathrm{C}$ under vacuum to the constant weight, leading to a light pink powder with a weight increase of $37.2 \%$ in comparison with "living" PMAA microspheres.

PMAA@P(MAA-co-BAC) microspheres (entry 7 in Table 1) were prepared according to the following procedure: To a one-neck round-bottom flask $(100 \mathrm{~mL})$ were added "living" PMAA microspheres $(60.47 \mathrm{mg})$ and dried acetonitrile $(60 \mathrm{~mL})$. After the above mixture was ultrasonically dispersed at room temperature for $2 \mathrm{~min}, \mathrm{MAA}(82 \mu \mathrm{L}, 0.96 \mathrm{mmol}), \mathrm{BAC}(83.18$ $\mathrm{mg}, 0.32 \mathrm{mmol})$, and AIBN (0.42 $\mathrm{mg}, 2.56 \mu \mathrm{mol})$ were added successively. The reaction mixture was then degassed by bubbling argon for $30 \mathrm{~min}$ in an ice-water bath, sealed, immersed into a thermostated oil bath at $60^{\circ} \mathrm{C}$, and magnetically stirred for $48 \mathrm{~h}$. After centrifugation, the resulting solid product was thoroughly washed with acetonitrile and then dried at $40{ }^{\circ} \mathrm{C}$ under vacuum to the constant weight, leading to a light pink powder with a weight increase of $39.5 \%$ in comparison with the starting "living" PMAA microspheres.

PMAA@P(NIPAAm-co-MBA) microspheres (entry 8 in Table 1) were prepared as follows: To a one-neck round-bottom flask $(250 \mathrm{~mL})$ were added "living" PMAA microspheres (150.58 $\mathrm{mg})$ and dried acetonitrile $(150 \mathrm{~mL})$. After the above mixture was ultrasonically dispersed at room temperature for $2 \mathrm{~min}$, NIPAAm (60.77 mg, $0.54 \mathrm{mmol})$, MBA (20.41 mg, $0.13 \mathrm{mmol}$ ), and AIBN $(0.21 \mathrm{mg}, 1.28 \mu \mathrm{mol})$ were added successively. The reaction mixture was then degassed by bubbling argon for $30 \mathrm{~min}$ in an ice-water bath, sealed, immersed into a 
thermostated oil bath at $60{ }^{\circ} \mathrm{C}$, and magnetically stirred for $24 \mathrm{~h}$. After centrifugation, the resulting solid product was thoroughly washed with acetonitrile and then dried at $40{ }^{\circ} \mathrm{C}$ under vacuum to the constant weight, leading to a light pink powder with a weight increase of $12.8 \%$ in comparison with the starting "living" PMAA microspheres.

\section{Preparation of "Living" PMAA Nanospheres with Surface-Bound Dithioester Groups}

(Entry 11 in Table 1). "Living" PMAA nanospheres with surface-bound dithioester groups were prepared via RAFTPP following our previously reported method. ${ }^{21}$ MAA $(1.33 \mathrm{~mL}, 15.60$ mmol), CDB (6.06 mg, $22.25 \mu \mathrm{mol})$, dried acetonitrile (125 mL), and AIBN (1.83 mg, 11.07 $\mu \mathrm{mol})$ were added into a one-neck round-bottom flask $(250 \mathrm{~mL})$ with an oval-shaped stirring bar (with a length of $3.0 \mathrm{~cm}$ ) inside successively. A clear purple solution was obtained after the above mixture was magnetically stirred at room temperature for $10 \mathrm{~min}$, which was then purged with argon for $30 \mathrm{~min}$ in an ice-water bath, sealed, immersed into a thermostated oil bath at $70{ }^{\circ} \mathrm{C}$, and stirred for $6 \mathrm{~h}(200 \mathrm{rpm})$. The resulting polymer particles were collected by centrifugation and subsequently washed with acetonitrile thrice. After being dried at $40{ }^{\circ} \mathrm{C}$ under vacuum to the constant weight, a light pink powder was obtained in a yield of $10 \%$.

To obtain enough "living" PMAA nanospheres for the following study, the above polymerization was repeated several times and the resulting products were mixed together.

\section{Preparation of "Living” Core-Shell-Structured PMAA@P(MAA-co-AnHEMA-co-}

\section{BAC) Nanospheres (Entry 13 in Table 1). PMAA@P(MAA-co-AnHEMA-co-BAC)} nanospheres (entry 13 in Table 1) were prepared as follows: To a one-neck round-bottom flask $(250 \mathrm{~mL})$ were added "living” PMAA nanospheres $(150.23 \mathrm{mg})$ and dried acetonitrile $(150 \mathrm{~mL})$. After the above mixture was ultrasonically dispersed at room temperature for $5 \mathrm{~min}$, AnHEMA (40.19 mg, $120.20 \mu \mathrm{mol})$, MAA (205 $\mu \mathrm{L}, 2.41 \mathrm{mmol}), \mathrm{BAC}(208.76 \mathrm{mg}, 0.80 \mathrm{mmol})$, and AIBN (1.10 mg, $6.67 \mu \mathrm{mol})$ were added successively. The reaction mixture was then degassed by bubbling argon for $30 \mathrm{~min}$ in an ice-water bath, sealed, immersed into a thermostated oil bath at $60{ }^{\circ} \mathrm{C}$, and magnetically stirred for $48 \mathrm{~h}$. After centrifugation, the resulting solid product was thoroughly washed with acetonitrile and then dried at $40{ }^{\circ} \mathrm{C}$ under vacuum to the constant weight, leading to a light pink powder with a weight increase of $25.5 \%$ in comparison with "living" PMAA nanospheres. 

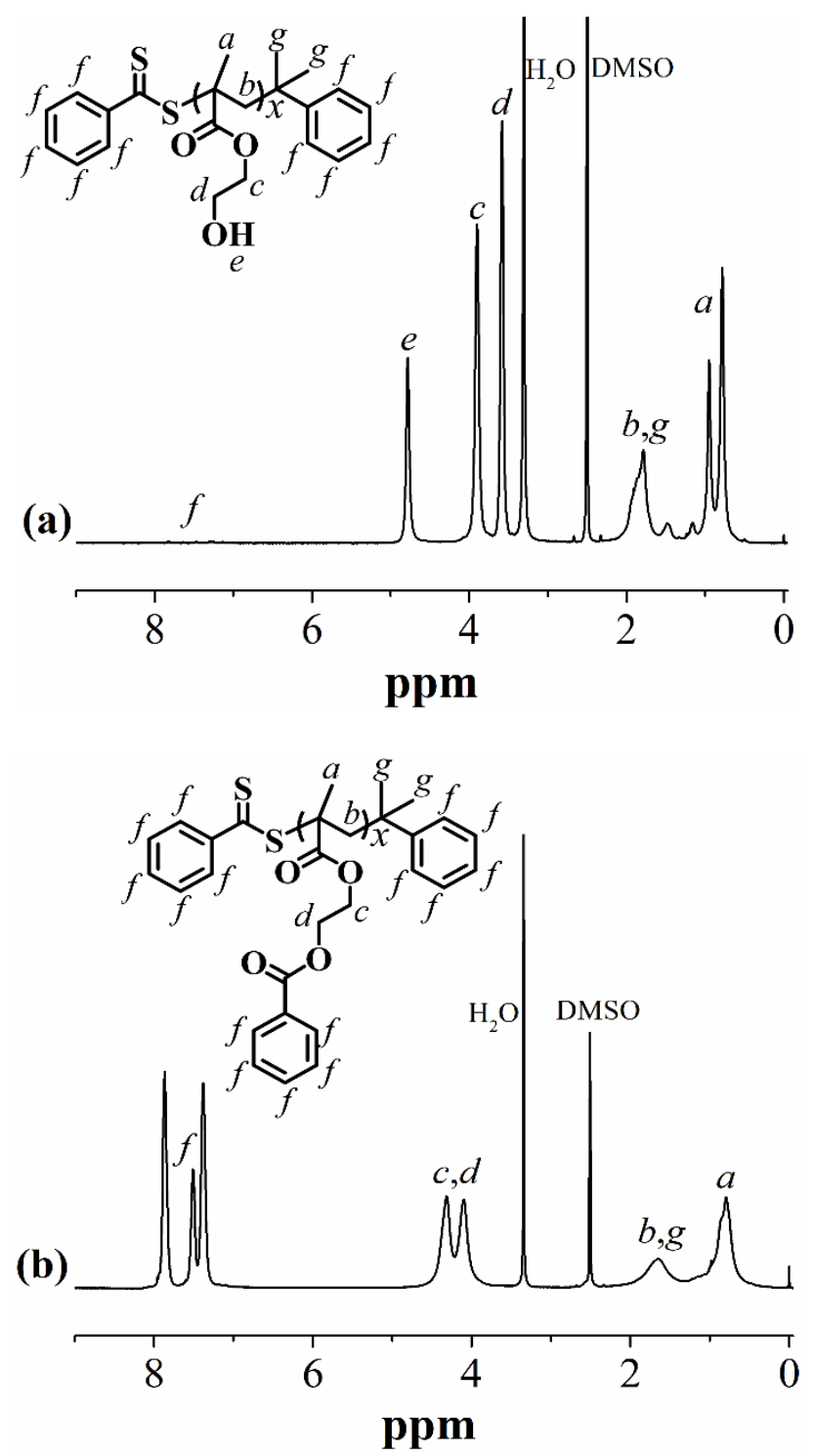

Figure S1. ${ }^{1} \mathrm{H}$ NMR spectra of one representative PHEMA (a) and its esterified polymer (with benzoic anhydride) (b) in the deuterated DMSO.

Figure S1a,b shows the ${ }^{1} \mathrm{H}$ NMR spectra of one representative PHEMA (which was generated in the polymerization solution during the preparation of PMAA@P(MAA-co-EGDMA)3@PHEMA (entry 9 in Table 1) via SI-RAFT polymerization of HEMA) and its esterified polymer (with benzoic anhydride) in DMSO-d6, respectively. It can be seen clearly that $100 \%$ of esterification was achieved for the PHEMA, as revealed by the total disappearance of the proton peak of the hydroxyl group (i.e., peak $e$ in Figure S1a) and the complete shift of the methylene proton peaks (peaks $c, d$ ) as well as the appearance of some very strong aromatic protons (peaks $f$ ) in the spectrum of the esterified PHEMA (Figure S1b). 
(a)

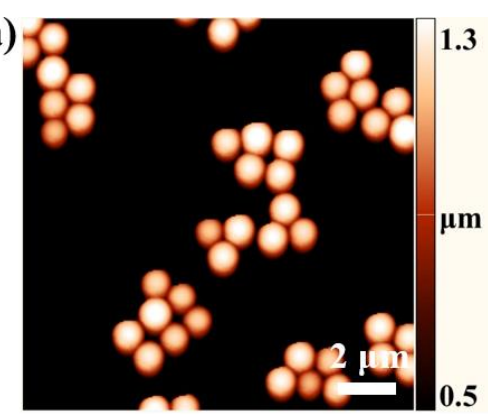

(b)

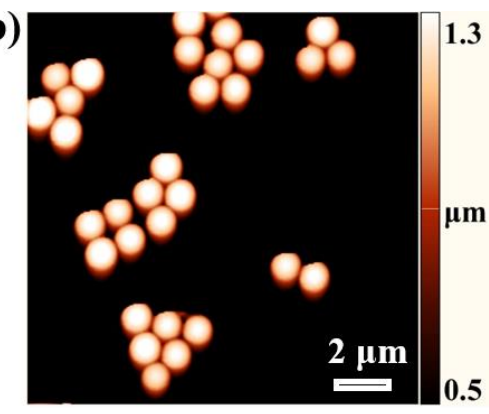

(c)

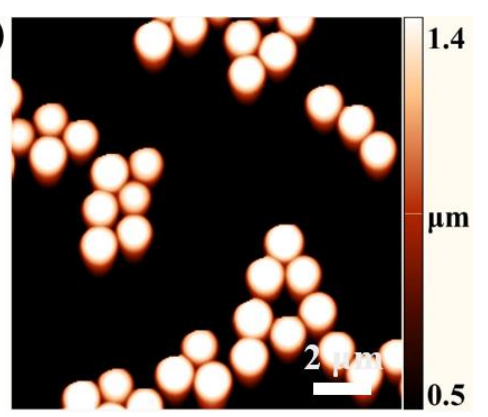

Figure S2. AFM height images of core-shell-structured polymer microspheres including PMAA@P(MAA-co-EGDMA)-1 PMAA@P(MAA-co-EGDMA)-2

(b), and PMAA@P(MAA-co-EGDMA)-4 (c) (samples a, b, and c correspond to entries 2, 3, and 5 in Table 1, respectively).

(a)

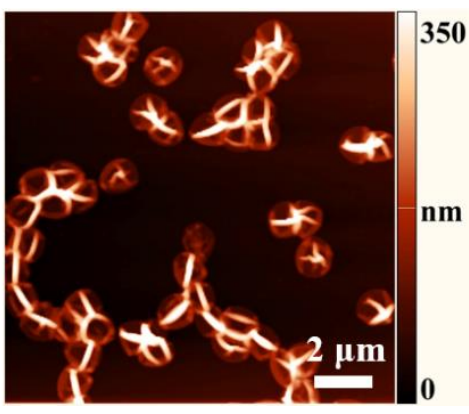

(b)

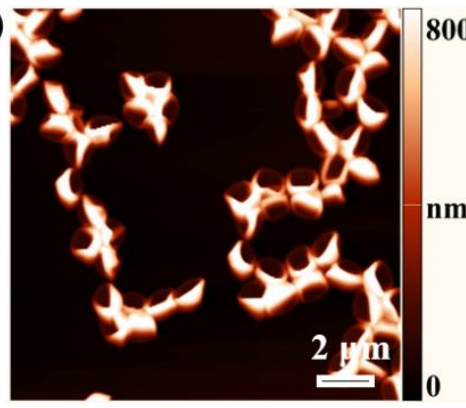

$800(c)$

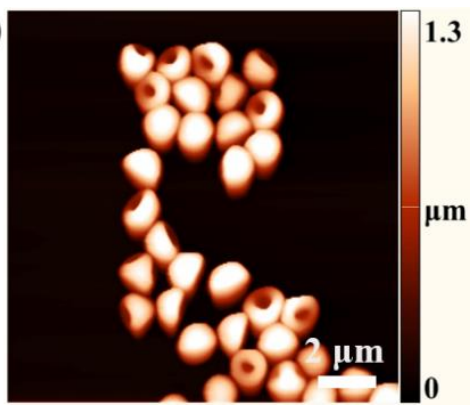

Figure S3. AFM height images of hollow polymer microparticles including H@P(MAA-coEGDMA)-1 (a), H@P(MAA-co-EGDMA)-2 (b), and H@P(MAA-co-EGDMA)-4 (c) (samples $\mathrm{a}, \mathrm{b}$, and c correspond to entries 1,2 , and 4 in Table 2 , respectively).

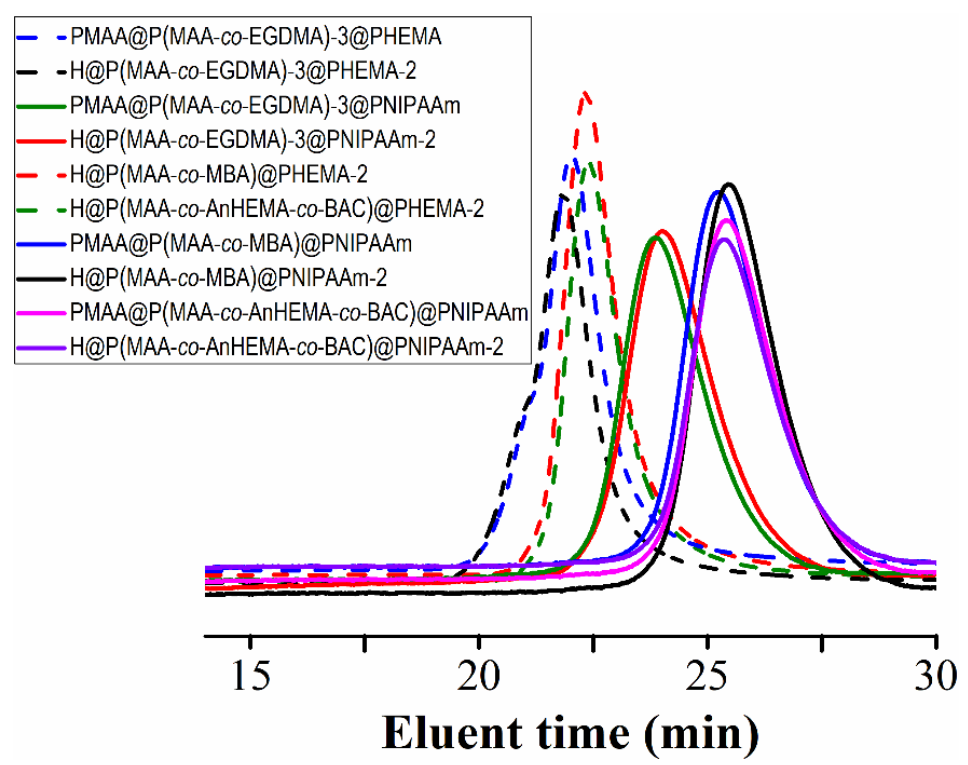

Figure S4. GPC traces of the free polymers generated in the SI-RAFT polymerization systems during the surface-grafting of hydrophilic polymer brushes onto solid core-shell-structured polymer particles and hollow polymer particles. 
Table S1. Number-average molecular weights $\left(M_{\mathrm{n}, \mathrm{GPC}}\right)$ and molar-mass dispersities $(\nexists)$ of the surface-grafted polymer brushes (determined by GPC) on polymer particles ${ }^{a}$

\begin{tabular}{clcc}
\hline Entry $^{b}$ & \multicolumn{1}{c}{ Sample } & $M_{\mathrm{n}, \mathrm{GPC}}$ & $\oplus$ \\
\hline 1 & PMAA@P(MAA-co-EGDMA)-3@PHEMA & 44400 & 1.14 \\
2 & PMAA@P(MAA-co-EGDMA)-3@PNIPAAm & 17500 & 1.19 \\
3 & H@P(MAA-co-EGDMA)-3@PHEMA-1 & 44400 & 1.14 \\
4 & H@P(MAA-co-EGDMA)-3@PNIPAAm-1 & 17500 & 1.19 \\
5 & H@P(MAA-co-EGDMA)-3@PHEMA-2 & 47600 & 1.13 \\
6 & H@P(MAA-co-EGDMA)-3@PNIPAAm-2 & 18400 & 1.17 \\
$\mathbf{7}$ & PMAA@P(MAA-co-MBA)@PNIPAAm & 12100 & 1.11 \\
$\mathbf{8}$ & PMAA@P(MAA-co-AnHEMA-co-BAC)@PNIPAAm & 11100 & 1.13 \\
$\mathbf{9}$ & H@P(MAA-co-MBA)@PNIPAAm-1 & 12100 & 1.11 \\
$\mathbf{1 0}$ & H@P(MAA-co-AnHEMA-co-BAC)@PNIPAAm-1 & 11100 & 1.13 \\
$\mathbf{1 1}$ & H@P(MAA-co-MBA)@PNIPAAm-2 & 10500 & 1.21 \\
$\mathbf{1 2}$ & H@P(MAA-co-AnHEMA-co-BAC)@PNIPAAm-2 & 10800 & 1.19 \\
$\mathbf{1 3}$ & H@P(MAA-co-MBA)@PHEMA-2 & 30700 & 1.22 \\
$\mathbf{1 4}$ & H@P(MAA-co-AnHEMA-co-BAC)@PHEMA-2 & 28900 & 1.25 \\
\hline
\end{tabular}

${ }^{a}$ Determined by characterizating the molecular weights and molar-mass dispersities of the free polymers generated in the SI-RAFT polymerization systems during the surface-grafting of polymer brushes onto "living" polymer particles; ${ }^{b}$ Entries 1-2 and 7-8 refer to core-shellcorona-structured polymer microspheres and nanospheres, respcetively, and entries 3-6 and 9-14 refer to hydrophilic hairy hollow polymer microparticles and nanoparticles, respectively.

The absolute number-average molecular weights of PHEMA and PNIPAAm were measured by ${ }^{1} \mathrm{H}$ NMR (Bruker Avance III $400 \mathrm{MHz}$ NMR spectrometer). Figure S5a,b presents the representative ${ }^{1} \mathrm{H}$ NMR spectra of PHEMA and PNIPAAm, respectively, from which the number-average molecular weights of the polymers $\left(M_{\mathrm{n}, \mathrm{NMR}}\right)$ were determined by using the following formulas (Tables 1 and 2): 


$$
M_{\mathrm{n}, \mathrm{NMR}, \mathrm{PHEMA}}=5 \times\left(S_{\mathrm{d}} / S_{\mathrm{f}}\right) \times M_{\mathrm{HEMA}}+M_{\mathrm{CDB}} \quad(\text { Formula } 1)
$$

where $S_{\mathrm{d}}$ and $S_{\mathrm{f}}$ refer to the integral of the peak $d$ around $3.60 \mathrm{ppm}$ and that of the peaks $f$ between 7 and 8 ppm, respectively, $M_{\text {HEMA }}$ the molar mass of HEMA, and $M_{\mathrm{CDB}}$ the molar mass of CDB.

$$
M_{\mathrm{n}, \mathrm{NMR}, \mathrm{PNIPAAm}}=10 \times\left(S_{\mathrm{c}} / S_{\mathrm{f}}\right) \times M_{\mathrm{NIPAAm}}+M_{\mathrm{CDB}} \quad(\text { Formula } 2)
$$

where $S_{\mathrm{c}}$ and $S_{\mathrm{f}}$ refer to the integral of the peak $c$ around $3.85 \mathrm{ppm}$ and that of the peaks $f$ between 7 and 8 ppm, respectively, $M_{\text {NIPAAm }}$ the molar mass of NIPAAm, and $M_{\mathrm{CDB}}$ the molar mass of CDB.
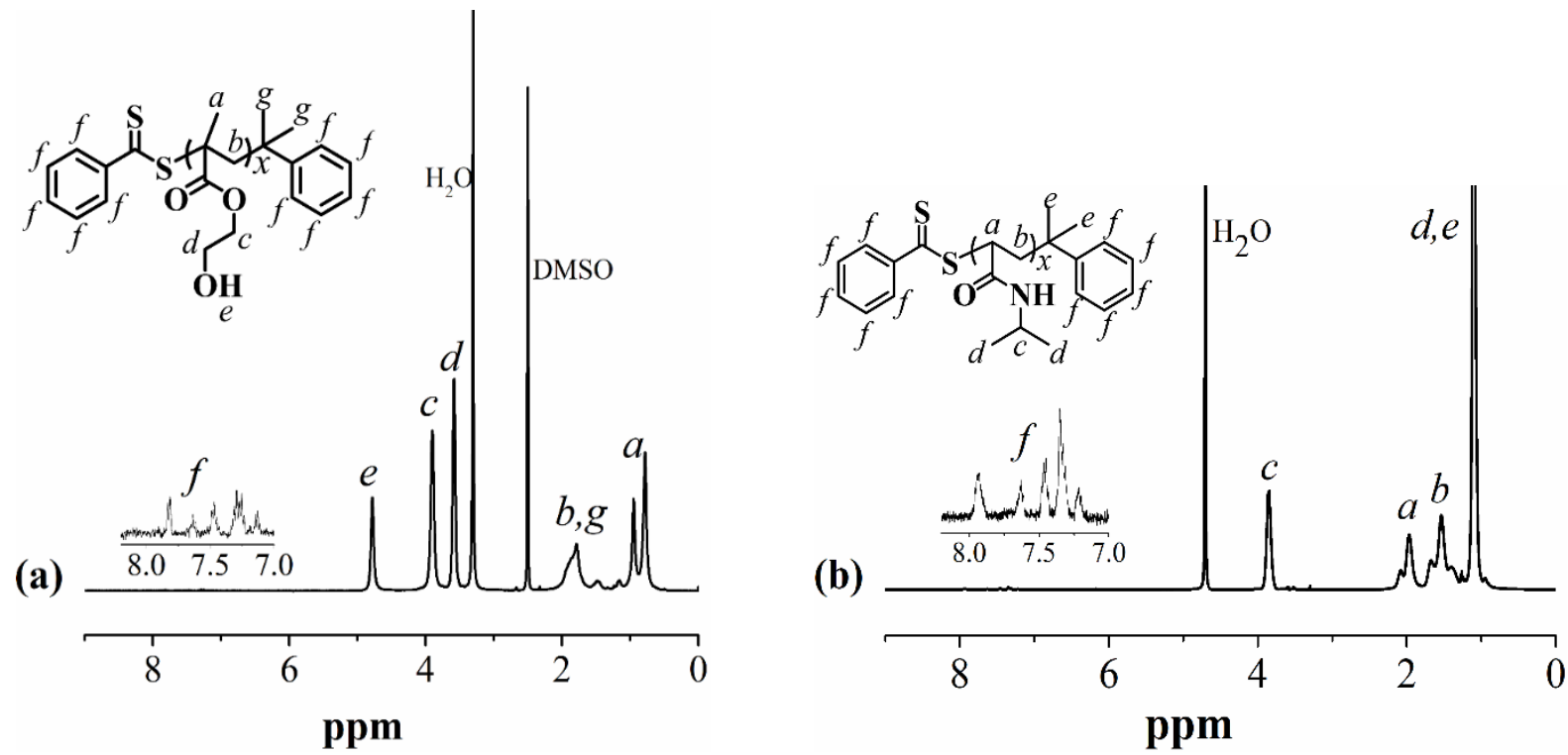

Figure S5. ${ }^{1} \mathrm{H}$ NMR spectra of the representative free polymers including PHEMA (in DMSOd6) (a) and PNIPAAm (in the deuterated water) (b) generated in the SI-RAFT polymerization systems during the preparation of PMAA@P(MAA-co-EGDMA)-3@PHEMA (entry 9 in Table 1) and PMAA@P(MAA-co-EGDMA)-3@PNIPAAm (entry 10 in Table 1) (or hydrophilic polymer brushes on H@P(MAA-co-EGDMA)-3@PHEMA-1 (entry 8 in Table 2) and H@P(MAA-co-EGDMA)-3@PNIPAAm-1 (entry 9 in Table 2)). 
(a)

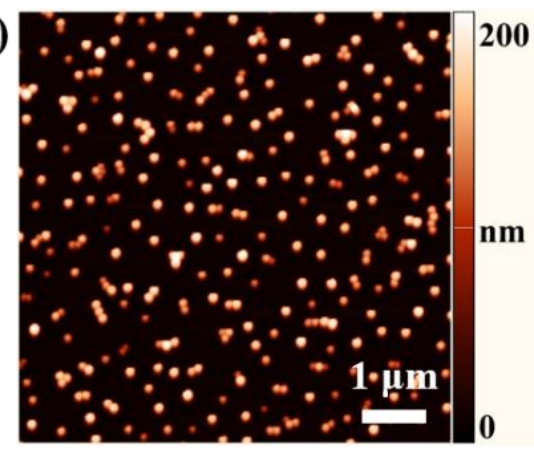

(b)

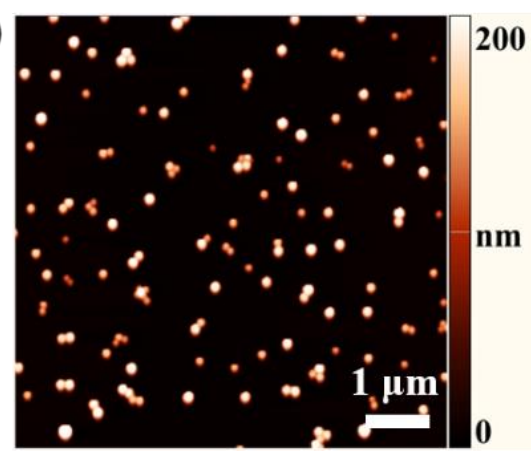

(c)

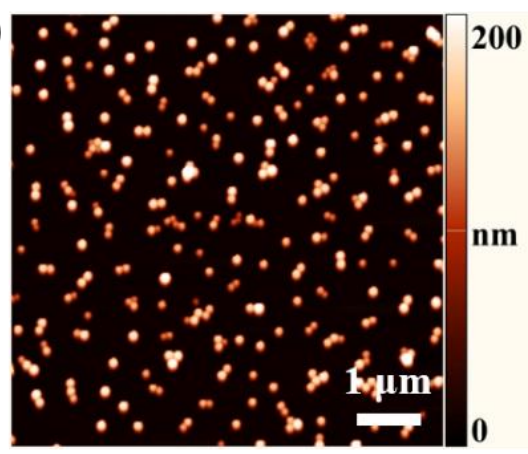

(d)

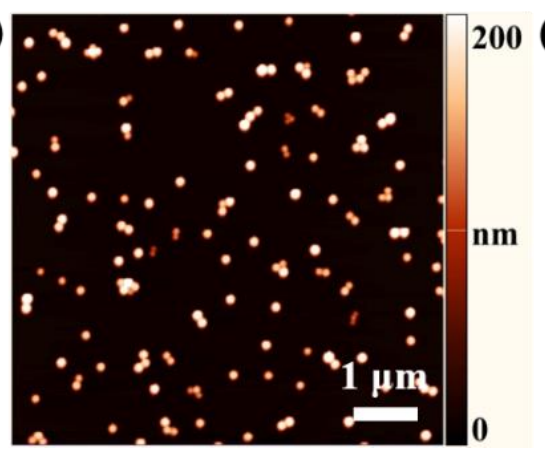

(e)

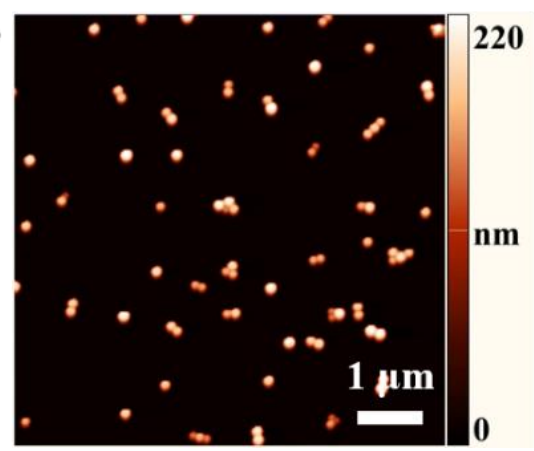

Figure S6. AFM height images of "living" PMAA nanospheres (a), core-shell-structured polymer nanospheres including PMAA@P(MAA-co-MBA) (b) and PMAA@P(MAA-coAnHEMA-co-BAC) (c), and core-shell-corona-structured polymer nanospheres including PMAA@P(MAA-co-MBA)@PNIPAAm (d) and PMAA@P(MAA-co-AnHEMA-coBAC)@PNIPAAm (e) (samples a, b, c, d, and e correspond to entries 11, 12, 13, 14, and 15 in Table 1, respectively). 


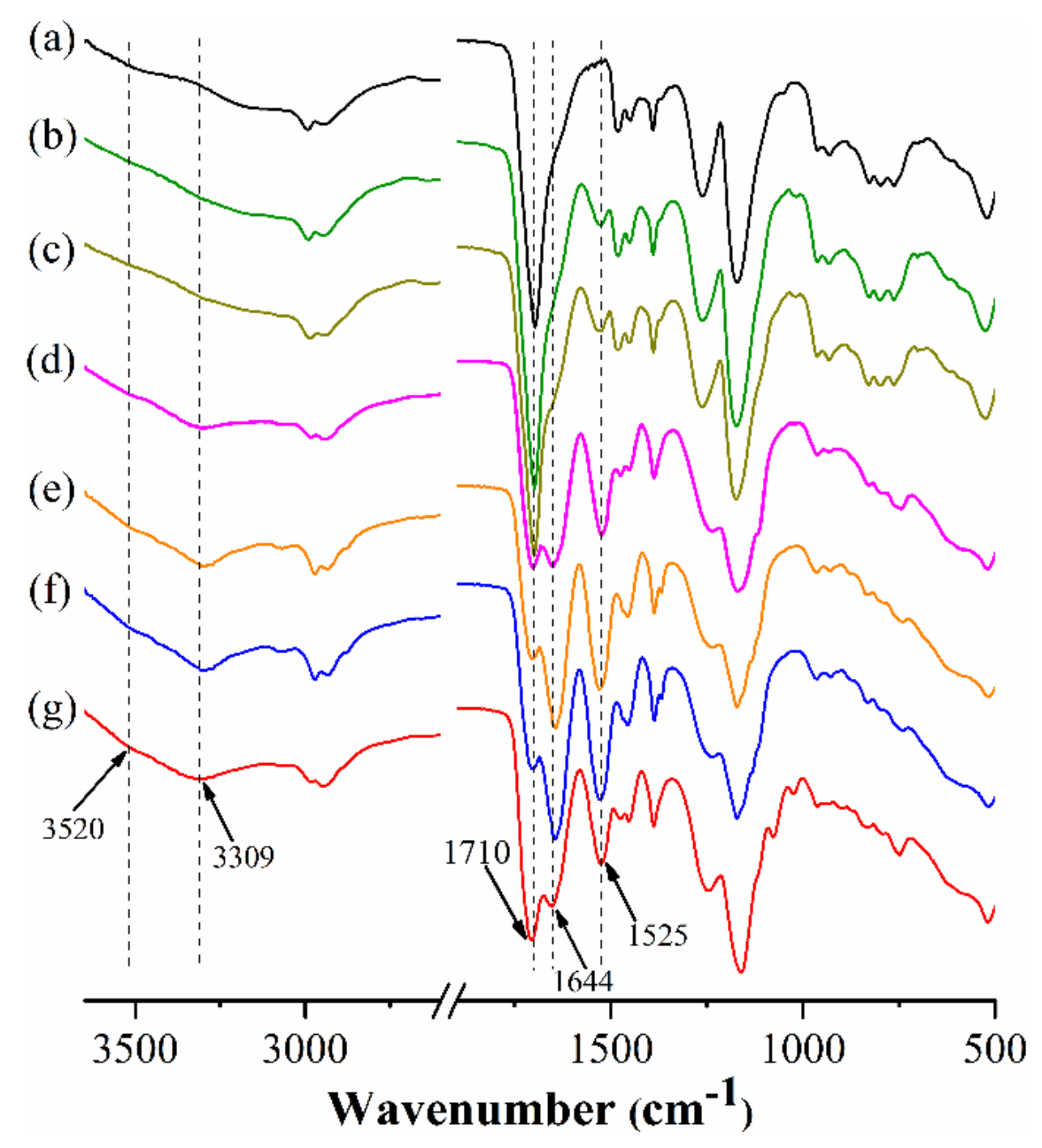

Figure S7. FT-IR spectra of "living" PMAA nanospheres (a), core-shell-structured polymer nanospheres PMAA@P(MAA-co-MBA) (b), core-shell-corona-structured polymer nanospheres PMAA@P(MAA-co-MBA)@PNIPAAm (c), hollow polymer nanoparticles H@P(MAA-co-MBA) (d), hairy hollow polymer nanoparticles prepared via Route 1 (i.e., H@P(MAA-co-MBA)@PNIPAAm-1) (e), and hairy hollow polymer nanoparticles prepared via Route 2 including H@P(MAA-co-MBA)@PNIPAAm-2 (f) and H@P(MAA-coMBA)@PHEMA-2 (g) (samples a, b, and c correspond to entries 11, 12, and 14 in Table 1, respectively, and samples $\mathrm{d}$, e, f, and g correspond to entries 12, 14, 16, and 18 in Table 2, respectively). 


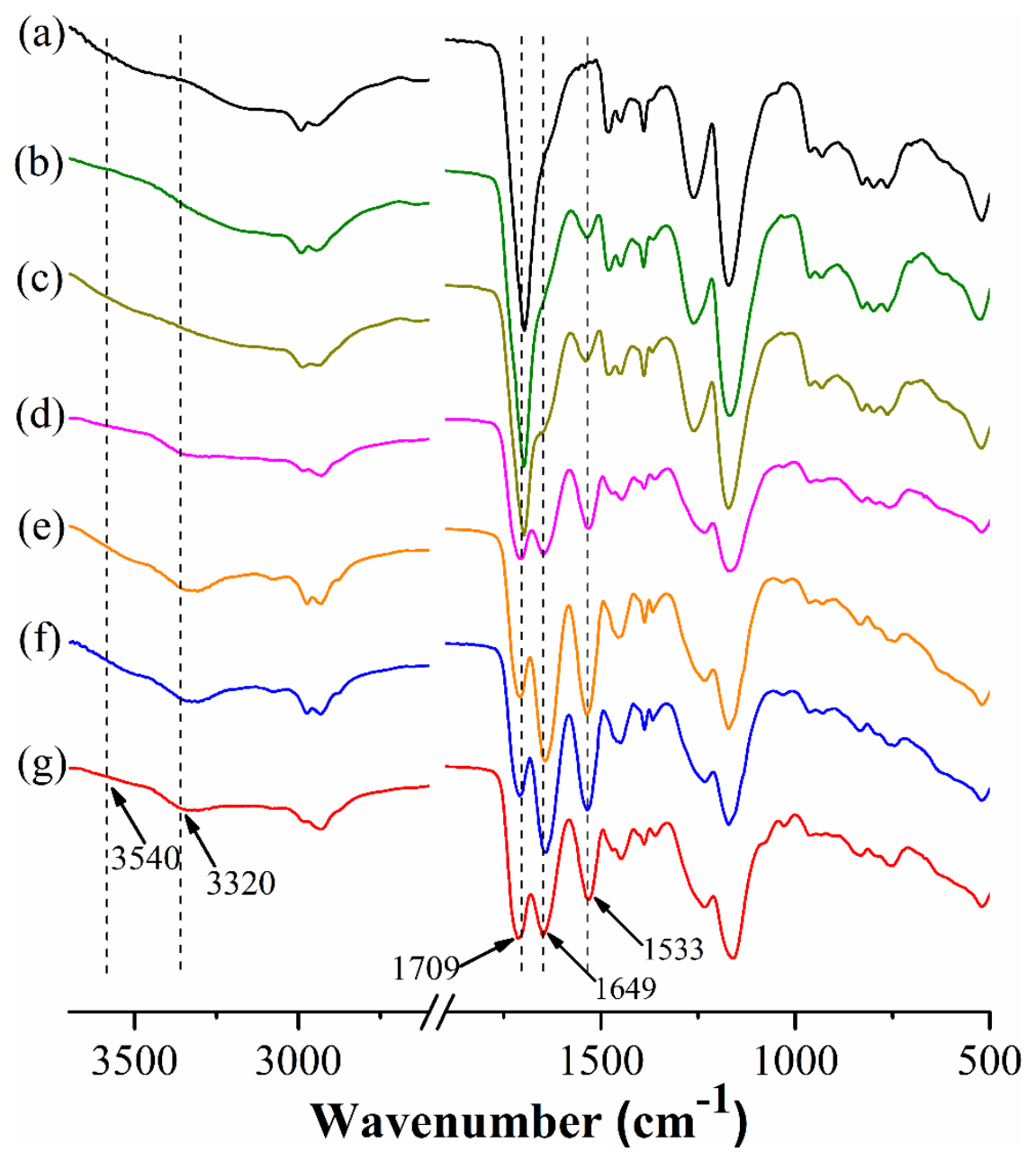

Figure S8. FT-IR spectra of "living" PMAA nanospheres (a), core-shell-structured polymer nanospheres PMAA@P(MAA-co-AnHEMA-co-BAC) (b), core-shell-corona-structured polymer nanospheres PMAA@P(MAA-co-AnHEMA-co-BAC)@PNIPAAm (c), hollow polymer nanoparticles H@P(MAA-co-AnHEMA-co-BAC) (d), hairy hollow polymer nanoparticles prepared via Route 1 (i.e., H@P(MAA-co-AnHEMA-co-BAC)@PNIPAAm-1) (e), and hairy hollow polymer nanoparticles prepared via Route 2 including H@P(MAA-coAnHEMA-co-BAC)@PNIPAAm-2 (f) and H@P(MAA-co-AnHEMA-co-BAC)@PHEMA-2 (g) (samples a, b, and c correspond to entries 11, 13, and 15 in Table 1, respectively, and samples $\mathrm{d}, \mathrm{e}, \mathrm{f}$, and $\mathrm{g}$ correspond to entries $13,15,17$, and 19 in Table 2, respectively). 


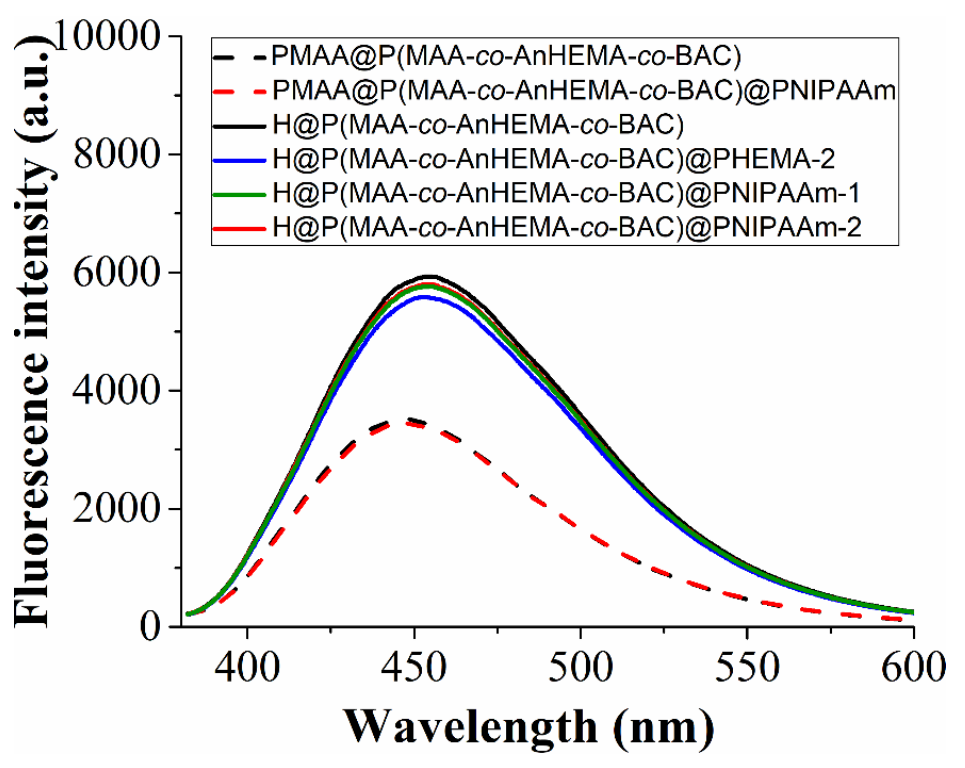

Figure S9. Fluorescent spectra of the dispersed mixtures of the solid core-shell- and core-shellcorona-structured polymer nanospheres with incorporated anthracene units in the shells in acetonitrile $\left(1.0 \mathrm{mg} \mathrm{mL}^{-1}\right)$ and those of hollow and hairy hollow polymer nanoparticles with incorporated anthracene units in the shells in methanol $\left(1.0 \mathrm{mg} \mathrm{mL}^{-1}\right.$ (corresponding to coreshell-corona particles before etching)). The fluorescent spectra of the samples were determined by using an F-4600 spectrofluorometer (Hitachi, Japan) with the excitation wavelength being $350 \mathrm{~nm}$ (EX slit width: $10 \mathrm{~nm}$; EM slit width: $10 \mathrm{~nm}$; voltage: $450 \mathrm{~V})$.

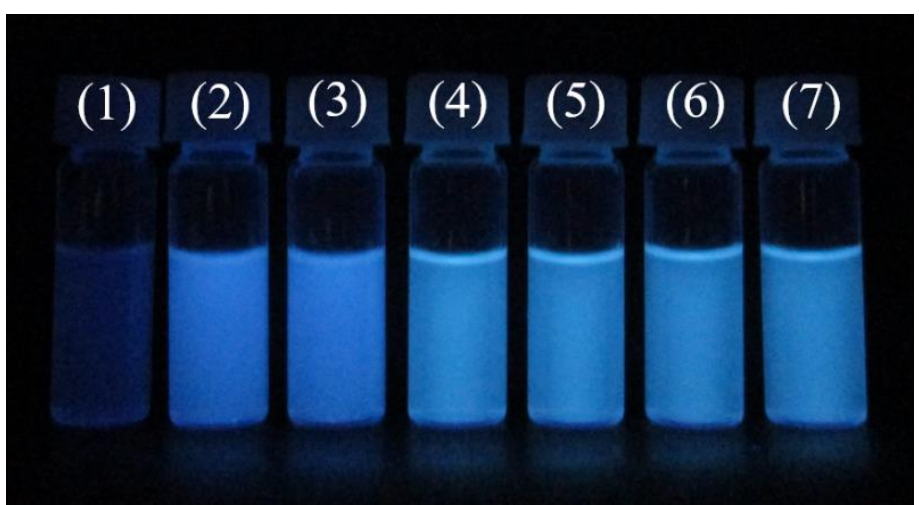

Figure S10. Photographs of the homogeneously dispersed mixtures of "living" PMAA nanospheres (1), core-shell-structured PMAA@P(MAA-co-AnHEMA-co-BAC) nanospheres (2), and core-shell-corona-structured PMAA@P(MAA-co-AnHEMA-co-BAC)@PNIPAAm nanospheres (3) in acetonitrile $\left(1.0 \mathrm{mg} \mathrm{mL}^{-1}\right)$ and those of hollow and hairy hollow polymer nanoparticles including H@P(MAA-co-AnHEMA-co-BAC) (4), H@P(MAA-co-AnHEMAco-BAC)@PHEMA-2 (5), H@P(MAA-co-AnHEMA-co-BAC)@PNIPAAm-1 (6), and H@P(MAA-co-AnHEMA-co-BAC)@PNIPAAm-2 (7) nanospheres in methanol (1.0 mg mL ${ }^{1}$, corresponding to solid particles before etching) under $365 \mathrm{~nm}$ UV light irradiation. 